\title{
CONSTRAINED MAX-MIN BANDWIDTH ALLO- CATION FOR THE AVAILABLE BIT RATE ATM SERVICE
}

\author{
Seung Hyong Rhee and Takis Konstantopoulos * \\ Department of Electrical \& Computer Engineering \\ University of Texas at Austin \\ Austin, Texas 78712, U.S.A. \\ \{shrhee,takis\} @alea.ece.utexas.edu
}

\begin{abstract}
The available bit rate (ABR) is an ATM service category that provides an economical support of connections having vague requirements. An ABR session may specify its peak cell rate (PCR) and minimum cell rate (MCR), and available bandwidth is allocated to competing sessions based on the max-min policy. In this paper, we investigate the ABR traffic control from a different point of view: Based on the decentralized bandwidth allocation model studied in [9], we prove that the max-min rate vector is the equilibrium of a certain system of noncooperative optimizations. This interpretation suggests a new framework for ABR traffic control that allows the max-min optimality to be achieved and maintained by end-systems, and not by network switches. Moreover, in the discussion, we consider the constrained version of max-min fairness and develop an efficient algorithm with theoretical justification to determine the optimal rate vector.
\end{abstract}

Keywords: ATM Networks, Available Bit Rate, Decentralized Control, Max-Min Fairness, Equilibrium

\section{INTRODUCTION}

The available bit rate (ABR) is an ATM service category that provides an economical support of connections having vague requirements. An end-system may specify both a peak cell rate (PCR) and a minimum cell rate (MCR) on the establishment of the ABR session, and varies its input rate exploiting the available bandwidth along its path.

\footnotetext{
*The authors of this work were supported in part by Grant ARP 224 of the Texas Higher Education Coordinating Board. The first author is now with Digital Communications Lab, Samsung Advanced Institute of Technology, P.O.Box 111, Suwon, 440-600, Korea.
} 
The ATM forum has proposed a rate-based closed-loop mechanism for the ABR traffic control: a network switch is responsible for computing a fair share of the available bandwidth among connections passing it and sending the information to end-systems. Available bandwidth is allocated to competing sessions based on the max-min policy, which has been widely used as a fairness criterion for bandwidth allocation [3]. Many algorithms have been suggested to implement this ABR control framework. For example, [11] proposed a scheme that uses Explicit Forward Congestion Indication (EFCI), which is a single bit indicator of congestion. To achieve a stable and speedy operation, explicit-rate feedback mechanisms were developed, for example, explicit rate indication [4] and uniform tracking [6].

In this paper, we investigate the ABR traffic control from a different point of view. Using the result on the decentralized bandwidth allocation model studied in [9], we prove that the max-min rate vector is the equilibrium of a certain system of noncooperative optimizations. This interpretation suggests a new framework for ABR traffic control that allows the max-min optimality to be achieved and maintained by end-systems, and not by network switches. In the framework, each session controller has a simple objective function that gives an optimal amount of bandwidth to be used for its connection, and dynamically adjusts its bandwidth responding to the link status along its path.

As ABR connections may specify their own minimum and maximum demands, the classical definition of max-min principle has been extended in the ABR traffic control literature to consider different MCR's or PCR's, e.g., maxmin allocation above the MCR [6] or adding one extra link per session to impose PCR [7]. Recently, in [8], the conventional max-min policy was generalized to the case of connections with different constraints on their demands, and conditions for the optimality was driven in the same fashion with the classical one. We develop an efficient algorithm to determine the max-min rate vector for the constrained case: Given the sessions' PCR's and MCR's, the algorithm directly computes the max-min rate vector, and is proved to finish in $K \cdot L$ steps, where $K$ and $L$ are the numbers of sessions and links, respectively.

This paper is organized as follows. The constrained version of max-min policy is introduced in Section 2. In Section 3, a system of noncooperative optimization problems is formulated and it is proved that its equilibrium achieves the constrained max-min fairness. The centralized computing algorithm is developed in Section 4, while Section 5 proposes a new decentralized framework for achieving max-min fairness and shows some simulation results. Finally, Section 6 concludes the paper with some remarks. 


\section{CONSTRAINED MAX-MIN POLICY}

Let $\mathcal{V}$ be a finite set of nodes and $\mathcal{L} \subseteq \mathcal{V} \times \mathcal{V}$ be a set of undirected links whose elements are unordered pairs of distinct nodes. Each link $\ell \in \mathcal{L}$ has an available bandwidth $C_{\ell}>0$. A set $\mathcal{K}=\{1, \ldots, K\}$ of sessions is given and share the network $\mathcal{G}=(\mathcal{V}, \mathcal{L})$. Session $i \in \mathcal{K}$ has a set $\mathcal{L}^{i} \subseteq \mathcal{L}$ of links that it is associated with. The links in $\mathcal{L}^{i}$ constitute a fixed path between two end points of session $i$. Moreover, it is assumed that session $i$ has Peak Cell Rate (PCR) $M^{i}$ and Minimum Cell Rate (MCR) $m^{i}$ such that $0 \leq m^{i} \leq M^{i}$. We denote the allocated rate (bandwidth) for session $i$ by $f^{i}$, and the rate vector for all sessions in $\mathcal{K}$ by $\mathbf{f}=\left(f^{1}, \ldots, f^{K}\right)$. The set of sessions crossing link $\ell$ can be defined by

$$
\mathcal{K}_{\ell}=\left\{i \in \mathcal{K} \mid \ell \in \mathcal{L}^{i}\right\},
$$

and the total rate at link $\ell$ is denoted as $F_{\ell}=\sum_{i \in \mathcal{K}_{\ell}} f^{i}$. Then, a rate vector $\mathrm{f}$ should satisfy the following constraints:

$$
\begin{array}{r}
m^{i} \leq f^{i} \leq M^{i} \quad \text { for all } \quad i \in \mathcal{K} \\
F_{\ell} \leq C_{\ell} \\
\text { for all } \quad \ell \in \mathcal{L} .
\end{array}
$$

The constrained version of max-min policy, which is a natural extension of the conventional one in [3], can be stated as follows [8]. A vector $\mathbf{f}$ is said to be feasible if the above constraints are satisfied. Moreover, a rate vector $\mathbf{f}$ is said to be max-min fair if it is feasible and, for each $i \in \mathcal{K}, f^{i}$ cannot be increased while maintaining feasibility without decreasing $f^{j}$ for some session $j$ for which $f^{j} \leq f^{i}$.

Definition 1 [8] Given a feasible rate vector $\mathbf{f}$, we say that link $\ell \in \mathcal{L}$ is a bottleneck link with respect to $\mathbf{f}$ for a session $i \in \mathcal{K}$ traversing $\ell$ if $F_{\ell}=C_{\ell}$ and $f^{i} \geq f^{j}$ for every session $j$ traversing link $\ell$ for which $f^{j}>m^{j}$.

In [3], using the conventional model of max-min fairness, i.e., without constraints, a necessary and sufficient condition for a feasible rate vector $\mathbf{f}$ to be max-min fair is given. It can be extended to the constrained case as follows:

Theorem 1 [8] A feasible rate vector $\mathbf{f}$ is max-min fair if and only if each session has either a bottleneck link with respect to $\mathrm{f}$ or a rate assignment equal to its maximum rate.

\section{ACHIEVING MAX-MIN FAIR RATE VECTOR}

In this section, we formulate a decentralized bandwidth allocation model and prove that its unique equilibrium corresponds to the max-min fair rate vector of the network. 


\subsection{A SYSTEM OF NONCOOPERATIVE OPTIMIZATIONS}

Assume that all sessions in $\mathcal{K}$ of network $\mathcal{G}=(\mathcal{V}, \mathcal{L})$ are active, and that each session independently decides an optimal rate for its $A B R$ connection according to the following optimization problem:

$$
\begin{array}{ll}
\operatorname{maximize} & \sum_{\ell \in \mathcal{L}^{i}} w_{\ell}^{i} \cdot U_{\ell}^{i}(\mathbf{f}), \quad i \in \mathcal{K} \\
\text { subject to } & m^{i} \leq f^{i} \leq M^{i} .
\end{array}
$$

The weighting factor $w_{\ell}^{i}$ is given by, for all $i$,

$$
w_{\ell}^{i}= \begin{cases}1, & \text { if } C_{\ell}^{i} \leq C_{\ell^{\prime}}^{i} \text { for all } \ell^{\prime} \in \mathcal{L}^{i} \\ 0, & \text { otherwise }\end{cases}
$$

where

$$
C_{\ell}^{i}=C_{\ell}-\sum_{j \neq \imath} f_{\ell}^{j}
$$

is the remaining bandwidth seen by session $i$ on link $\ell . U_{\ell}^{i}$ is a real function that is strictly concave with respect to $f^{i}$ given a remaining bandwidth $C_{\ell}^{i}$ and that has its optimal at

$$
\underset{f^{2}}{\arg \max } U_{\ell}^{i}(\mathbf{f})=\alpha C_{\ell}^{2}=\alpha\left(C-\sum_{j \neq i} f^{j}\right),
$$

where $\alpha$ is a real number such that $0<\alpha<1$. The system of optimizations given by (1) is a special case of the decentralized bandwidth allocation model which was studied in [9]. According to Theorem 2 in [9], which establishes the uniqueness of the Nash equilibrium [5] of the decentralized model, (1) also has a unique equilibrium at which point no session can increase its objective without decreasing other sessions' objectives.

We now analyze the bandwidth allocation $\mathrm{f}^{*}$ achieved by the equilibrium. The set $\mathcal{K}_{\ell}$ can be divided into two disjoint subsets

$$
\mathcal{K}_{\ell}^{1}=\left\{i \in \mathcal{K}_{\ell} \mid w_{\ell}^{* i}=1\right\} \quad \text { and } \quad \mathcal{K}_{\ell}^{0}=\left\{i \in \mathcal{K}_{\ell} \mid w_{\ell}^{* i}=0\right\} .
$$

The equilibrium bandwidth of session $i \in \mathcal{K}_{\ell}^{1}$ can be figured out by the lemma below.

Lemma 1 For a session $i \in \mathcal{K}$ that passes through link $\ell \in \mathcal{L}$ and $w_{\ell}^{i}=1$ at the equilibrium, its bandwidth is determined such as

$$
f^{* i}=\left\{\begin{aligned}
M^{i} & , \quad \text { if } M^{i}<F S_{\ell} \\
m^{i} & , \text { if } m^{i}>F S_{\ell} \\
F S_{\ell} & , \text { otherwise }
\end{aligned}\right.
$$


where the unique point $F S_{\ell}$ is given by

$$
F S_{\ell}=\frac{\alpha}{1-\alpha}\left(C_{\ell}-F_{\ell}^{*}\right) .
$$

Proof. Due to space limitations, we only sketch the idea of proof; Refer to the proof of Lemma 2 in [10] for details. Let $F S_{\ell}=\frac{\alpha}{1-\alpha}\left(C_{\ell}-F_{\ell}^{*}\right)$. Then, by the Kuhn-Tucker conditions [2] at the equilibrium, we obtain the following implications:

$$
\begin{aligned}
& M^{i}<F S_{\ell} \Rightarrow f^{* i}<F S_{\ell} \Rightarrow \lambda^{* i}>\mu^{* i} \Rightarrow f^{* i}=M^{i} \\
& m^{i}>F S_{\ell} \Rightarrow f^{* i}>F S_{\ell} \Rightarrow \lambda^{* i}<\mu^{* i} \Rightarrow f^{* i}=m^{i},
\end{aligned}
$$

where $\lambda^{* i}$ and $\mu^{* i}$ are Lagrange multipliers. The remaining case $f^{* i}=F S_{\ell}$ can be proved by removing the users of the first two cases and computing the unconstrained equilibrium.

\subsection{MAX-MIN FAIRNESS OF THE EQUILIBRIUM}

All sessions in $\mathcal{K}_{\ell}$ can be divided further into five disjoint sets as Figure 1. A session with $w_{\ell}^{i}=1$ belongs to one of three sets $\mathcal{A}_{\ell}, \mathcal{B}_{\ell}$, and $\mathcal{K}_{\ell}^{\prime}$, which are defined by

$$
\begin{aligned}
\mathcal{A}_{\ell} & =\left\{i \in \mathcal{K}_{\ell}^{1} \mid m^{i}>F S_{\ell}\right\} \\
\mathcal{B}_{\ell} & =\left\{i \in \mathcal{K}_{\ell}^{1} \mid M^{i}<F S_{\ell}\right\} \\
\mathcal{K}_{\ell}^{\prime} & =\mathcal{K}_{\ell}^{1}-\mathcal{A}_{\ell}-\mathcal{B}_{\ell} .
\end{aligned}
$$

Session $i$ in $\mathcal{K}_{\ell}^{0}$ has a link $\ell^{\prime} \neq \ell$ along its path $\mathcal{L}^{i}$, and its equilibrium bandwidth is determined in link $\ell^{\prime}$ by Lemma 1 . However, in link $\ell$, sessions in $\mathcal{K}_{\ell}^{0}$ can be divided into two sets, $\left\{i \in \mathcal{K}_{\ell}^{0} \mid f^{* i} \geq F S_{\ell}\right\}$ and $\left\{i \in \mathcal{K}_{\ell}^{0} \mid f^{* i}<F S_{\ell}\right\}$, depending on the value of $F S_{\ell}$.

Then, the total flow in link $\ell$ at the equilibrium is

$$
F_{\ell}^{*}=\sum_{i \in \mathcal{A}_{\ell}} m^{i}+\sum_{i \in \mathcal{B}_{\ell}} M^{i}+\left|\mathcal{K}_{\ell}^{\prime}\right| \cdot F S_{\ell}+\sum_{i \in \mathcal{K}_{\ell}^{0}} f^{* i} .
$$

If we substitute (3) into (2) and let $\alpha \rightarrow 1$, the limiting value of $F S_{\ell}$ is

$$
\lim _{\alpha \rightarrow 1} F S_{\ell}=\frac{1}{\left|\mathcal{K}_{\ell}^{\prime}\right|}\left(C_{\ell}-\sum_{i \in \mathcal{A}_{\ell}} m^{i}-\sum_{i \in \mathcal{B}_{\ell}} M^{i}-\sum_{i \in \mathcal{K}_{\ell}^{0}} f^{* i}\right), \quad \ell \in \mathcal{L} .
$$

Thus putting (4) in (3) yields

$$
\lim _{\alpha \rightarrow 1} F_{\ell}^{*}=C_{\ell}, \quad \ell \in \mathcal{L} .
$$

In the theorem below, it turns out that the allocation $\mathrm{f}^{*}$ by (1) becomes the max-min fair rate vector as $\alpha \rightarrow 1$. 


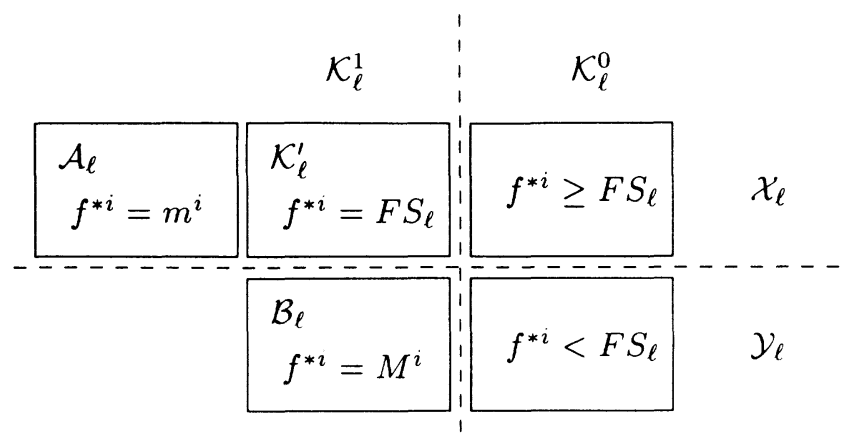

Figure 1 Five disjoint sets of sessions passing link $\ell$ at the equilibrium.

Theorem 2 As $\alpha \rightarrow 1$, the limit of bandwidth allocation $\mathrm{f}^{*}$ in the system of optimizations given by $(1)$ is the max-min fair rate vector of network $\mathcal{G}=(\mathcal{V}, \mathcal{L})$ for the sessions $\mathcal{K}$.

Proof. Clearly $\mathbf{f}^{*}$ is feasible, since $\mathbf{f}^{*} \in \mathcal{S}$ and $F_{\ell}^{*} \leq C_{\ell}$ for all $\ell$ [9]. We consider a link $\ell$ and examine all five disjoint subsets in Figure 1. First, for a session $i \in \mathcal{K}_{\ell}^{\prime}$ or $i \in \mathcal{A}_{\ell}, f^{* i}=F S_{\ell} \vee m^{i} \geq F S_{\ell}$. For any session $j$ for which $f^{* j}>m^{j}, j \in \mathcal{K}_{\ell}$,

$$
f^{* j}\left\{\begin{aligned}
=F S_{\ell} \wedge M^{i} \leq F S_{\ell} & , \text { if } \quad j \in \mathcal{K}_{\ell}^{1} \\
<F S_{\ell} & , \text { if } j \in \mathcal{K}_{\ell}^{0} .
\end{aligned}\right.
$$

The second inequality comes from the fact that for a session in $\left\{i \in \mathcal{K}_{\ell}^{0} \mid f^{* i} \geq\right.$ $\left.F S_{\ell}\right\}, f^{* i}=m^{i}$, by Lemma 2 below. Thus for session $i$ in $\mathcal{K}_{\ell}^{\prime} \cup \mathcal{A}_{\ell} \cup\{i \in$ $\left.\mathcal{K}_{\ell}^{0} \mid f^{* i} \geq F S_{\ell}\right\}$,

$$
f^{* i} \geq F S_{\ell} \geq f^{* j} \text { for any } j \text { for which } f^{* j}>m^{j}, \quad j \in \mathcal{K}_{\ell} .
$$

At the limit of $\mathbf{f}^{*}$ as $\alpha \rightarrow 1, F_{\ell}^{*} \rightarrow C_{\ell}$. Then by Definition $1, \ell$ is a bottleneck link with respect to $\mathrm{f}^{*}$ for a session in $\mathcal{K}_{\ell}^{\prime} \cup \mathcal{A}_{\ell} \cup\left\{i \in \mathcal{K}_{\ell}^{0} \mid f^{* i} \geq F S_{\ell}\right\}$. Now consider a session $i \in\left\{i \in \mathcal{K}_{\ell}^{0} \mid f^{* i}<F S_{\ell}\right\}$. i has at least one link $\ell^{\prime} \neq \ell, \ell^{\prime} \in \mathcal{L}^{i}$, for which $w_{\ell^{\prime}}^{i}=1$. By the same reasoning, the session belongs to $\mathcal{K}_{\ell^{\prime}}^{\prime}, \mathcal{A}_{\ell^{\prime}}$ or $\mathcal{B}_{\ell^{\prime}}$. In case it belongs to $\mathcal{K}_{\ell^{\prime}}^{\prime}$ or $\mathcal{A}_{\ell^{\prime}}, \ell^{\prime}$ is its bottleneck link, otherwise $f^{* i}=M^{i}$. Thus each session passing link $\ell$ has a bottleneck link or a rate equal to its PCR. Thus by Theorem $1, \mathrm{f}^{*}$ is a max-min fair rate vector as $\alpha \rightarrow 1$. 
Lemma 2 Session $i$ in $\left\{i \in \mathcal{K}_{\ell}^{0} \mid f^{* i} \geq F S_{\ell}\right\}$ has a rate equal to its MCR at $\mathbf{f}^{*}$, i.e., $f^{* i}=m^{i}$.

Proof. As the session belongs to $\mathcal{K}_{\ell}^{0}$, there is a link $\ell^{\prime} \neq \ell, \ell^{\prime} \in \mathcal{L}^{i}$, such that $w_{\ell^{\prime}}^{i}=1$, i.e. $C_{\ell}^{* i}>C_{\ell^{\prime}}^{* i}$. Then for the session $i \in \mathcal{K}_{\ell}^{0}$ for which $f^{* i} \geq F S_{\ell}$,

$$
\begin{aligned}
f^{* i} & \geq F S_{\ell}=\frac{\alpha}{1-\alpha}\left(C_{\ell}-F_{\ell}^{*}\right)=\frac{\alpha}{1-\alpha}\left(C_{\ell}^{* i}-f^{* i}\right) \\
& >\frac{\alpha}{1-\alpha}\left(C_{\ell^{\prime}}^{* i}-f^{* i}\right)=\frac{\alpha}{1-\alpha}\left(C_{\ell^{\prime}}-F_{\ell^{\prime}}^{*}\right)=F S_{\ell^{\prime}}
\end{aligned}
$$

Thus $f^{* i}=m^{i}$ at link $\ell^{\prime}$ by Lemma 1 .

\section{COMPUTING ALGORITHM}

We develop a simple algorithm for computing the max-min fair rate vector f. The algorithm is formulated in the following way. First, using Algorithm 1 below which determines the fair share solution of a single link, we compute the solution for each link in the network. Then, it is iterated until the max-min rate vector of the network is found, by removing bottleneck link(s) at each iteration.

We are given the link capacity $C_{\ell}$ and information on sessions in $\mathcal{K}_{\ell}$, i.e., $M^{i}$,s and $m^{i}$ s. Algorithm 1, which is a modified version of Algorithm 1 in [10], computes $F S_{\ell}$ of link $\ell$, so that each sessions rate can be determined by Lemma 1. It is easy to see that $F S_{\ell}$ is determined in at most $\left|K_{\ell}\right|$ iterations.

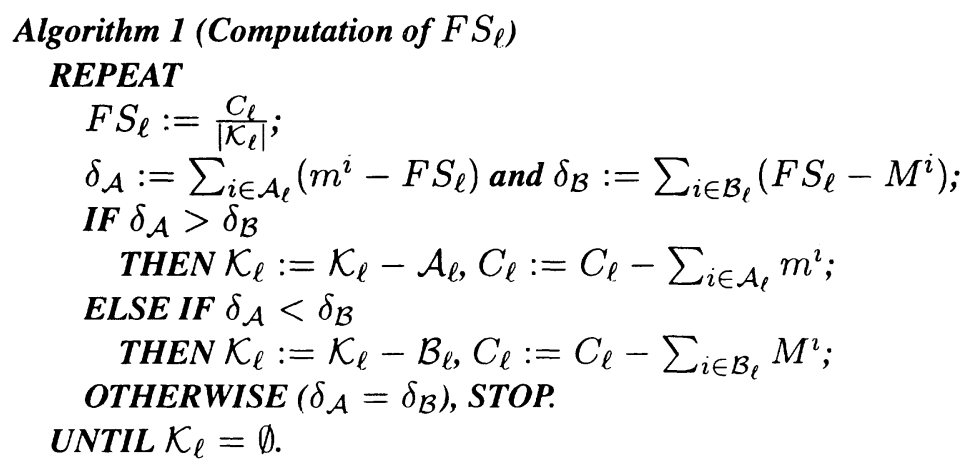

We now develop an algorithm for computing the max-min rate vector for network $\mathcal{G}=(\mathcal{V}, \mathcal{L})$. At each iteration of the algorithm, $F S_{\ell}$ is independently computed for all links using Algorithm 1. The idea is to find link $\ell$ such that $F S_{\ell} \leq F S_{\ell^{\prime}}$ for all $\ell^{\prime} \in \mathcal{L}$. If we let $p$ be such a link, then by virtue of Proposition 1 below, we can assure that $\mathcal{K}_{p}^{0}=\emptyset$ at the link. That is, $F S_{p}$ can be determined by only the local information at link $p$, and the sessions in $\mathcal{K}_{p}$ have rate assignments regardless of other links. 
Proposition 1 For a network $\mathcal{G}=(\mathcal{V}, \mathcal{L})$ and sessions in $\mathcal{K}$, let $p$ be the link for which $F S_{p}=\min _{\ell} F S_{\ell}$ for all $\ell \in \mathcal{L}$, where each $F S_{\ell}, \ell \in \mathcal{L}$, is independently computed at each link by Algorithm 1. Then

$$
w_{p}^{i}=1, \quad \text { for all } i \in \mathcal{K}_{p} .
$$

Proof. Consider a session $i$ in $\mathcal{K}_{p}$, and let $q$ be a link such that $q \neq p$ and $q \in \mathcal{L}^{i}$. Then by (2), for any $\alpha \in(0,1)$,

$$
\frac{\alpha}{1-\alpha}\left(C_{p}-F_{p}\right)=F S_{p} \leq F S_{q}=\frac{\alpha}{1-\alpha}\left(C_{q}-F_{q}\right) .
$$

Thus for any $i$ in $\mathcal{K}_{p}$,

$$
C_{p}^{i}=C_{p}-\sum_{\substack{j \neq i \\ j \in \mathcal{K}_{p}}} f^{j}=C_{p}-F_{p}+f^{\imath}<C_{q}-F_{q}+f^{\imath}=C_{q}-\sum_{\substack{j \neq i \\ j \in \mathcal{K}_{q}}} f^{\jmath}=C_{q}^{\imath} .
$$

At next iteration, the link(s) which has the smallest $F S_{\ell}$ is removed from $\mathcal{L}$, and the sessions crossing the link(s) is regarded as constant rate sessions; i.e., for session $i$ in $\mathcal{K}_{p}$, where $p$ is removed from the set of links, let $M^{i}=m^{i}=f^{i}$. The algorithm is iterated until all links are removed. Then clearly every session has a bottleneck link or rate assignment equal to its PCR. The max-min fairness follows by Theorem 1 .

\section{Algorithm 2 (max-min fair bandwidth allocation of network $\mathcal{G}$ ) REPEAT}

$$
\begin{aligned}
& \text { For each } \ell \in \mathcal{L} \text {, compute } F S_{\ell} \text { using Algorithm 1; } \\
& \mathcal{P}:=\left\{\ell \in \mathcal{L} \mid F S_{\ell} \leq F S_{\ell^{\prime}} \text { for all } \ell^{\prime} \in \mathcal{L}\right\} ; \\
& \quad / * \text { For sessions passing link }(s) \text { in } \mathcal{P}, f^{i} \text { is determined by }(1) . * / \\
& \text { Let } m^{i}:=M^{i}:=f^{i} \text { for } i \in \mathcal{K}_{\ell}, \ell \in \mathcal{P} \text {, and } \mathcal{L}:=\mathcal{L}-\mathcal{P} ; \\
& \text { UNTIL } \mathcal{L}=\emptyset .
\end{aligned}
$$

Since at least one link is removed form the set of links, Algorithm 2 terminates in at most $|\mathcal{L}|$ iterations. For a faster computation, $\mathcal{L}$ can be reduced further in step 3 by removing the link(s) that is not in $\mathcal{P}$ but has all constant rate sessions. This feature is not implemented in Algorithm 2, and its possible effect can be checked in the following example.

Example 1 The network configuration in Figure 2, which is borrowed from [1], consists of four switches connected via three links with capacity 100 each. Four constrained sessions are established and each link is shared by at least two sessions.

Table 1 shows the procedure for computing $\mathbf{f}=\left(f^{1}, f^{2}, f^{3}, f^{4}\right)$ using Algorithm 2. First, $F S_{\ell}, \ell=1, \ldots, 4$, is computed and $F S_{3}$ is the smallest. 


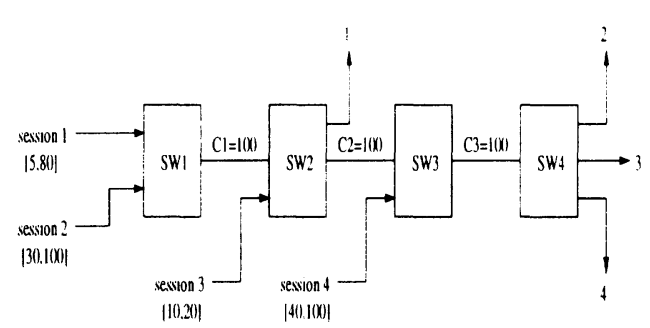

Figure 2 Network configuration of four switches connected via three links. Each session rate is constrained by its MCR and PCR which are denoted by two numbers in the bracket.

Thus we can assign rates to session 2,3 , and 4 according to Lemma 1. Those assigned rates will not be changed during the computation. At the next iteration, we consider only link 1 and link 2 , and $F S_{1}>F S_{2}$. However since all sessions in $\mathcal{K}_{2}$ were assigned rates at the first iteration, nothing is changed in the second iteration except the set $\mathcal{L}$. If we modify the algorithm so that link 2 is removed at the first iteration, then the algorithm terminates in two iterations. Finally, only link 1 is remained and $\mathbf{f}$ is determined as $(60,40,20,40)$. Its max-min fairness is asserted by Theorem 1 .

Table 1 Computation of max-min fair vector of the four-switch network using Algorithm 2.

\begin{tabular}{crrrrrrrrrr}
\hline iter & \multicolumn{3}{c}{ step 1 } & & step 2 & \multicolumn{5}{c}{ step 3 } \\
\cline { 2 - 6 } \cline { 7 - 9 } & $F S_{1}$ & $F S_{2}$ & $F S_{3}$ & $\mathcal{P}$ & $f^{1}$ & $f^{2}$ & $f^{3}$ & $f^{4}$ & $\mathcal{L}^{\prime}$ \\
\hline 1 & 50 & 80 & 40 & $\{3\}$ & & 40 & 20 & 40 & $\{1,2\}$ \\
\hline 2 & 60 & 50 & & $\{2\}$ & & 40 & 20 & 40 & $\{1\}$ \\
\hline 3 & 60 & & & $\{1\}$ & 60 & 40 & 20 & 40 & $\emptyset$ \\
\hline
\end{tabular}

\section{DECENTRALIZED FRAMEWORK FOR ABR TRAFFIC CONTROL}

In this section we suggest a new decentralized framework to achieve the maxmin fair bandwidth allocation based on the results of Section 3. We consider a dynamic scheme in which each ABR session adjusts its rate dynamically in response to network status. Let $\mathbf{f}(t)$ be a $K$-dimensional vector whose components are session rates at a discrete time $t$. Then the dynamics of the 
system can be formulated by the following iterative equation:

$$
\mathbf{f}(t+1)=T(\mathbf{f}(t)), \quad t=0,1, \ldots,
$$

where $T$ is some continuous mapping. Sessions change their rates iteratively until $\mathbf{f}$ converges to the fixed point solution of (5), according to the following rule.

Session behavior If session $i \in \mathcal{K}$ has a chance to update its rate at time $t$, it changes its rate such as, given remaining capacities of links in $\mathcal{L}^{i}$,

$$
f^{i}(t)=M^{i} \wedge\left[\alpha C_{p}^{i}(t) \vee m^{i}\right],
$$

where $p \in \mathcal{L}^{i}$ is a link such that $C_{p}^{i}(t) \leq C_{q}^{i}(t)$ for all $q \in \mathcal{L}^{i}$.

Note that response of a session given by (6) is the optimal of the optimization problem given in (1). Thus, if $\alpha$ is close to 1 , the fixed point solution of the above system corresponds to the equilibrium of (1) which has the max-min property.

We adopt the Gauss-Seidel type iteration by which only one component of $f$ can be updated at a time and the most recent information is available. Thus, in our implementation, $\mathbf{f}(t-1)$ and $\mathbf{f}(t)$ can be different only in their $i^{t h}$ element. A couple of assumptions are used in this framework. First, we do not specify how sessions obtain the information on the link status: We assume that sessions are able to acquire the information from the switches or by measurement. Second, it is assumed that there is no information delay.

The distinct feature of this scheme is that it achieves the max-min fairness by the sessions' independent and simple computations as in (6). Network switches do not perform any traffic control, nor maintain any local information. Moreover, the rate adjust mechanism given by (6) guarantees that there is no overflow at any time as long as MCR's are all zeros.

The Gauss-Seidel type algorithm can be implemented in two different ways: In a synchronous implementation, sessions update their rate in a specified order. On the other hand, there is no specified order in an asynchronous one. In the following subsection, we present simulation results using the asynchronous iteration.

\subsection{ASYNCHRONOUS ITERATIONS}

There are many ways to select a session at each time, for example, first-comefist-serve based on requests of sessions or random selection among sessions.

Figure 3 shows the changes in individual and total rates in a single link network with $C=100$. With $\alpha=0.99$, the individual rates converge to the fair share rate of the link. Each session has an opportunity to change its rate 

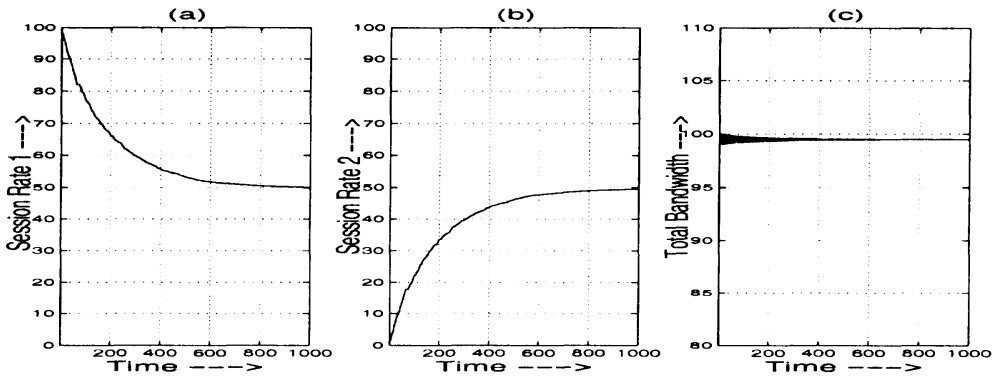

Figure 3 Asynchronous iterations in the single link with two unconstrained sessions: (a) session rate 1 (b) session rate 2 (c) total rate
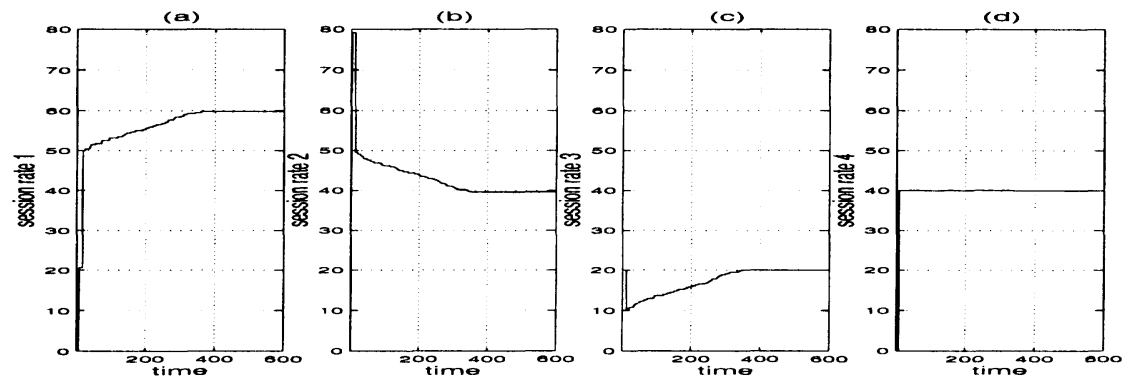

Figure 4 Changes of four individual session rates in the four-switch network by the asynchronous iterations
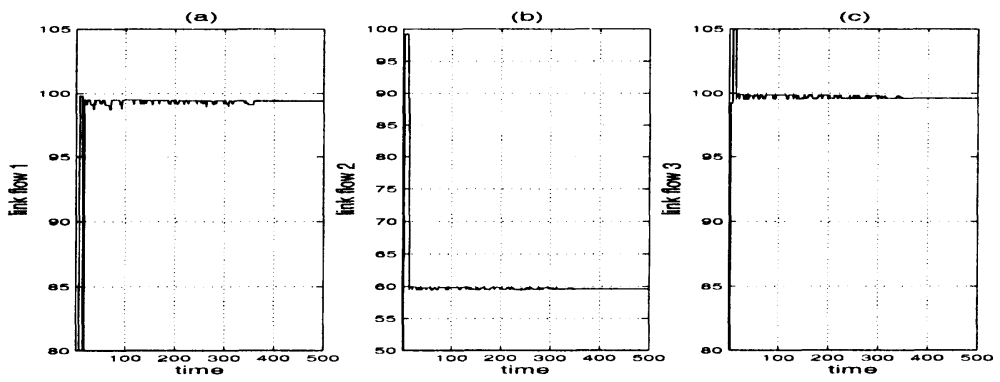

Figure 5 Total link rates in the asynchronous iterations

with equal probability. As session rates are changed in non-periodic manners, graphs of Figure 3 show irregular fashions.

Similarly, the asynchronous iteration can be applied to general topology networks with constrained sessions. Figure 4 and Figure 5 depict the rate 
changes of individual sessions and links, respectively, in the four switch network of Figure 2. With $\alpha=0.99$, four individual rates converge to $(60,40,20,40)$ as computed in Example 1.

\subsection{SPEEDING UP THE CONVERGENCE RATE}

If $K$ unconstrained sessions share a single link, at any value of $\alpha$ they equally share the capacity at the steady-state of the iterations. From (2) the rate of a session will be

$$
f^{i}=\frac{\alpha}{1-\alpha}(C-F)=\frac{\alpha}{1-\alpha}\left(C-K f^{i}\right)=\frac{\alpha}{1+(K-1) \alpha} C
$$

and the total flow should be

$$
F=K f^{\imath}=\frac{K \alpha}{1+(K-1) \alpha} C
$$

In Figure 3, as we set $\alpha=0.99$, the actual rates at the steady-state are $f^{1}=$ $f^{2}=49.75$ and $F=99.5$. In order to let the residual capacity shrink to zero, $\alpha$ should be very close to 1 . The problem here is that, as $\alpha \rightarrow 1$, it takes very long time for the rates to converge to the steady-state. Figure (6) shows the effect of $\alpha$ : As $\alpha$ goes to 1, total rate in the link increases, however it takes much more time to be converged.

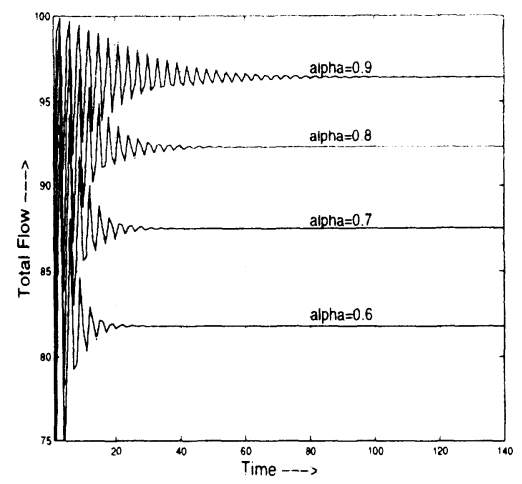

Figure 6 The effect of $\alpha$ on the convergence of the algorithm. As $\alpha$ goes to 1 , the total flow increases with lower converging speed.

One approach to avoid the difficulty is to give a value of $\alpha$ so that a target utilization of the link is achieved. Given target utilization $\rho$ and capacity $\bar{C}$, to achieve the total rate $\rho \bar{C}$, the value of $\alpha$ is determined from (7) such as

$$
\alpha=\frac{F}{K \bar{C}-(K-1) F}=\frac{\rho}{K-(K-1) \rho} .
$$


Note that the value of $\alpha$ to achieve $\rho$, depends only on $K$, and is independent of $\bar{C}$. Figure 7 depicts this approach for $\rho=0.9$ in a single link with $\bar{C}=100$. As $K=2, \alpha$ is set to $\frac{\rho}{2-\rho}=0.8182$ by (8). Individual session rates achieve the equal share rate 45 very fast, and the exact link utilization is attained as desired.
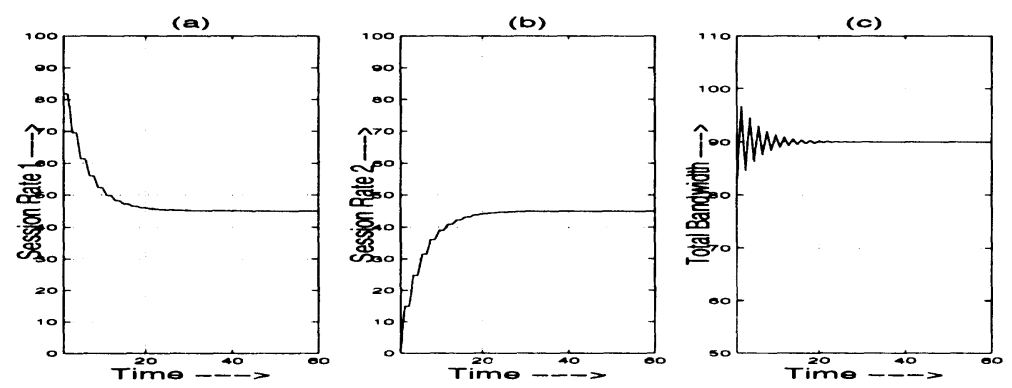

Figure 7 Achieving max-min bandwidth allocation by link utilization $\rho$. Target link utilization $C=90$ is attained at the steady state: (a) session rate 1 (b) session rate 2 (c) total flow

\section{CONCLUSIONS}

We have considered the constrained version of max-min bandwidth allocation for the ABR traffic control in ATM networks. Based on the decentralized bandwidth allocation model of [9], we proved that the equilibrium of a certain system of noncooperative optimizations is the max-min rate vector, and develop an efficient algorithm to determine the vector. The above work suggested a new framework for ABR traffic control such that the max-min fairness can be achieved by simple interactions of ABR sessions not by network switches. Simulation results were presented using asynchronous Gauss-Seidel type iteration.

It should be noted that the decentralized framework proposed in this paper awaits investigation on the effect of information delay to the convergence properties of the algorithm.

\section{References}

[1] Arulambalam, A., Chen, X., and Ansari, N. (1996). Allocating fair rates for available bit rate service in ATM networks. IEEE Communications Magazine, pages 92-100.

[2] Bertsekas, D. (1995). Nonlinear Programming. Athena Scientific, Belmont, MA. 
[3] Bertsekas, D. and Gallager, R. (1992). Data Networks. Prentice Hall, London, 2nd edition.

[4] Charny, A., Clark, D., and Jain, R. (1995). Congestion control with explicit rate indication. In Proceedings of IEEE ICC, pages 1954-1963.

[5] Fudenberg, D. and Tirole, J. (1992). Game Theory. MIT Press, Cambridge, MA.

[6] Fulton, C., Li, S.-Q., and Lim, C. S. (1997). An ABR feedback control scheme with tracking. In Proceedings of IEEE INFOCOM, pages 805814.

[7] Gafni, E. and Bertsekas, D. (1984). Dynamic control of session input rates in communication networks. IEEE Trans. on Automatic Control, 29(11):1009-1016.

[8] Hou, Y., Tzeng, H., and Panwar, S. (1997). A generalized max-min network capacity assignment policy with a simple ABR implementation for an ATM LAN. In Proceedings of IEEE GLOBECOM, pages 503-508.

[9] Rhee, S. H. and Konstantopoulos, T. (1999a). A decentralized model for virtual path capacity allocation. In Proceedings of IEEE INFOCOM, pages 497-504, NY.

[10] Rhee, S. H. and Konstantopoulos, T. (1999b). Decentralized optimal flow control with constrained source rates. IEEE Communications Letters, 3(6): 188-190.

[11] Yin, N. and Hluchyj, M. (1994). On closed-loop rate control for ATM cell relay networks. In Proceedings of IEEE INFOCOM, pages 99-108. 